\title{
Detection Method of Human Three-Dimensional Body Center of Gravity Based on Inclinometer Network
}

\author{
Jin-Zhuang Xiao, ${ }^{*}$ Zhi-Fang Yang, Hong-Rui Wang, and Xin-Cai Yang ${ }^{1}$ \\ College of Electronic and Information Engineering, Hebei University, \\ No. 180, Wusi East Road, Baoding, Hebei 071000, China \\ ${ }^{1}$ Healthy Rehabilitation Section, Affiliated Hospital of Hebei University, \\ No. 212, Yuhua East Road, Baoding, Hebei 071000, China
}

(Received April 1, 2016; accepted June 19, 2017)

Keywords: center of gravity, inclinometer network, motion capture system, segmental kinematic method, balance function

Research on the center of gravity (COG) of the body has wide applications in academic, medical, and other related fields. However, common devices are bulky, expensive, or inconvenient. In addition, most devices detect a two-dimensional center of mass, not a three-dimensional (3D) one. In this research, we proposed a detecting method of 3D COG. Consideration of body balance processes was realized by the rotation of each joint, and the processes could be detected by an inclinometer network installed on each main joint. On the basis of data from this network, we deduced a 3D-coordinate formula for the human COG during a tilted state using an analytical method and coordinate transformation. Then, the 3D coordinates of the COG were obtained by substituting tilt angles into the formula. Twenty subjects participated in the test. Compared with the results from a commercial motion capturing system, the standard deviation (SD) was in the range of $0.10-0.60 \mathrm{~cm}$, which validates the accuracy of the proposed system. The system is convenient, economical, and requires fewer calculations.

\section{Introduction}

The COG is an imaginary point where the total body gravity is concentrated. The trajectory of the COG during movement has been investigated, as its vertical displacement is an indicator of a body's movement, which can provide useful information about balance.

Many methods have been proposed to assess the position of the center of mass (COM: the same as COG). Some are based on the segmental kinematic centroid (SKC) method, ${ }^{(1)}$ while others are based on the mean of force platforms, which is still regarded as the gold standard in the analysis of movement or running. ${ }^{(2)}$ Some simple methods are also used, such as the sacrum or reconstructed pelvis methods. However, the intended segmental method is considered a better method for detecting the human COG, because it is more accurate than either the sacrum or reconstructed pelvis method. Although the force platform can also detect the location of COG in individuals who are walking or standing, it cannot be used to measure an entire COG oscillation in complex actions such as running, jumping, or whenever the subject has to face external forces during an aerial maneuver. Conversely, the SKC method appears to be appropriate to accomplish this task.

*Corresponding author: e-mail: robot@hbu.edu.cn

http://dx.doi.org/10.18494/SAM.2017.1592

ISSN 0914-4935 C MYU K.K. 
It regards the structure of the body as a set of rigid bodies, and their positions in time and space are detected using a motion capture system. The Songjing model also provides inertial parameters of the body segments, and R. Tisserand also suggested that the estimation of COM with 13 markers is very close to estimations based on 38 markers. ${ }^{(3,4)}$ Therefore, on the basis of this model, the position of the COG can be calculated as the centroid of a multisegment system. However, the SKC method is mainly based on a capture system; this method increases the complexity of computations, and the capture system is expensive and cannot be easily used in a clinic. The starting point of this study is to provide a useful and simple tool for COG estimation based on an inclinometer network.

This paper is organized as follows. In Sect. 2, we state the main theory and details of the experiments on this method. In Sect. 3, we present an evaluation of the results. In Sect. 4, we summarize the discussion and results. In Sect. 5, we briefly summarize the conclusions of the study.

\section{Materials and Methods}

First, in this study, we introduce data from the Songjing model, according to which the human body consists of fifteen segments and the positions of the COG of those fifteen segments are obtained. On the basis of the SKC model, we deduced the coordinates of a three-dimensional (3D) gravity center equation, Eq. (1), where $M$ is the weight, $m_{i}$ is the weight of each segment, $m_{i} / M$ is the mass ratio, ${ }^{(5-7)}$ and $x_{i}$ is the COM of the main segment of the human body.

$$
\begin{aligned}
& x_{\text {com }}=\frac{1}{M} \sum_{i=1}^{m} x_{i} m_{i}=\sum_{i=1}^{m} x_{i} \frac{m_{i}}{M} \\
& y_{\text {com }}=\frac{1}{M} \sum_{i=1}^{m} y_{i} m_{i}=\sum_{i=1}^{m} y_{i} \frac{m_{i}}{M} \\
& z_{\text {com }}=\frac{1}{M} \sum_{i=1}^{m} z_{i} m_{i}=\sum_{i=1}^{m} z_{i} \frac{m_{i}}{M}
\end{aligned}
$$

For ease of calculation, in this study, we consider that a human body consists of five segments, namely, two sides of upper limbs, two sides of lower limbs, and the trunk; therefore, $m=5$. Based on data from the Songjing model, the mass ratio of the five composite segments is 0.0505 (two sides of lower limbs), 0.1725 (trunk), and 0.555 (two sides of upper limbs). ${ }^{(8)}$ In summary, if the coordinates of the five segments' COG in 3D are obtained, then the 3D coordinates of the COG of an individual would be obtained.

During a tilting process, the angles of pitch and roll can be obtained using an inclinometer network system (INS) and coordinate conversion; then the coordinates of five segments may be determined. Equation (1) is then replaced by Eq. (2), where ${ }^{O} A,{ }_{B},{ }^{O} C$, ${ }^{O} D$, and ${ }^{O} E$ are the coordinates of the $\mathrm{COM}$ of the five segments in the original static state, which are combined based on the percentage of the length between the proximal and distal endpoints of the five segments. ${ }^{(9)}$ The term ${ }_{P}^{O} R(P=A, B, C, D, E)$ represents the spin matrix of the five segments during tilting, described in Eq. (3). The terms $\alpha_{i}$ and $\beta_{i}$ represent the rotation angle of each segment in the anteroposterior and lateral directions. 


$$
\begin{aligned}
& {\left[\begin{array}{l}
x_{\text {com }} \\
y_{\text {com }} \\
z_{\text {com }}
\end{array}\right]=\left[\begin{array}{c}
\sum_{i=1}^{m} x_{i} \frac{m_{i}}{M} \\
\sum_{i=1}^{m} y_{i} \frac{m_{i}}{M} \\
\sum_{i=1}^{m} z_{i} \frac{m_{i}}{M}
\end{array}\right] } \\
&= 0.1725\left({ }^{O} A_{1}+{ }^{O} B_{1}\right)+0.555^{O} C_{2}+0.0505\left({ }^{O} D_{3}+{ }^{O} E_{3}\right) \\
&= 0.1725\left({ }_{A}^{O} R^{O} A+{ }_{B}^{O} R^{O} B\right)+0.555_{C}^{O} R^{O} C_{1}+0.0505\left({ }_{D}^{O} R^{O} D_{2}+{ }_{E}^{O} R^{O} E_{2}\right) \\
&{ }_{P}^{O} R=R\left(x_{0}, \alpha_{i}\right) R\left(y_{0}, \beta_{i}\right)=\left[\begin{array}{ccc}
\cos \alpha_{i} \cos \beta_{i} & -\sin \alpha_{i} & \cos \alpha_{i} \sin \beta_{i} \\
\sin \alpha_{i} \cos \beta_{i} & \cos \alpha_{i} & \sin \alpha_{i} \sin \beta_{i} \\
-\sin \beta_{i} & 0 & \cos \beta_{i}
\end{array}\right]
\end{aligned}
$$

By using Eq. (2), this method provides a new detection system of an individual's 3D COG. The details of its structure are shown in Fig. 1.

During data collection, the five inclinometers were connected in series and installed at the position of the COG of the five segments. In this way, each segment's rotational angle could be measured in real time. Using acquired data in Eqs. (2) and (3), the 3D coordinate of the COG was obtained. In the measurement system, the CPU is a PC104 of Em104P-i2904, made by Arbor Technology Corp. The operating system is a QNX system, which is based on a microkernel. The detailed operation is described in Refs. 1 and 10.

To further validate the feasibility of the detecting method of 3D COG, we adopted the contrast experiment method, in which a motion capture system (MCS) was used as a reference group. It can follow the positions of markers pasted on the human body, and its detailed operation is described in Refs. 11 and 12. According to the Songjing model, the human body consists of fifteen parts; therefore, the subjects were marked with 32 markers. In this study, human bodies were defined by five segments, so five inclinometers were pasted at the location of each of the five main segments' COG; the details of the operation are shown in Fig. 2.

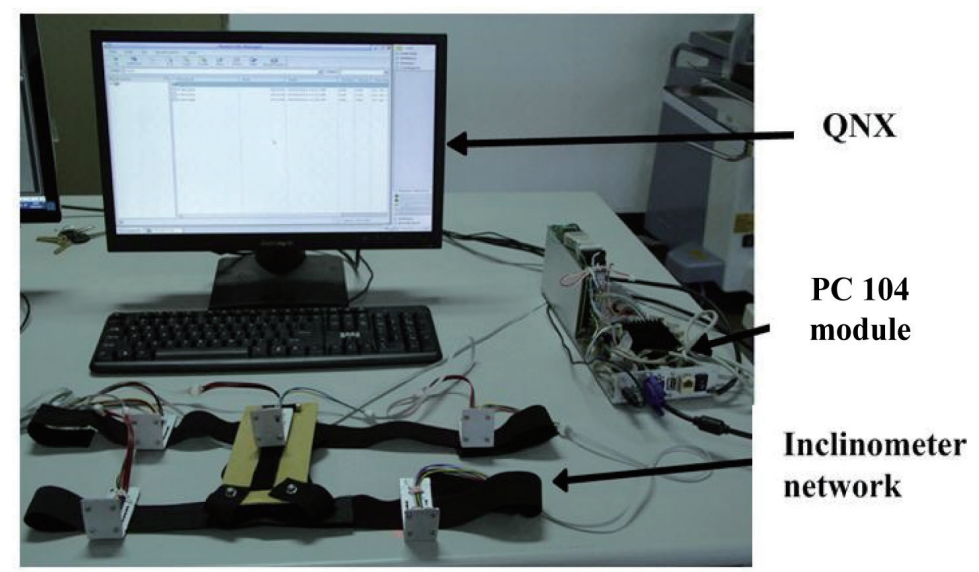

Fig. 1. (Color online) Experimental apparatus: The system consists of an inclinometer network, PC104, and a QNX operating system. The inclinometer system collected signals and transferred them to the PC104 module. The data were filtered, displayed, and stored in a QNX data file for further analysis. 

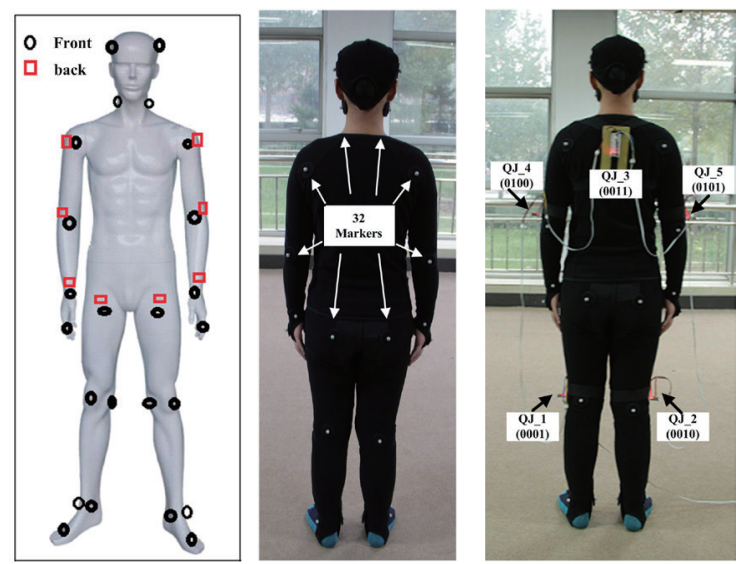

Fig. 2. (Color online) Positions of 32 markers, the locations of which are based on the Songjing model. By analytical methods, the position of the COG of five segments has been obtained.

Twenty healthy college students took part in the experiment and detected their center of gravity in two ways at the same time. After setting up the experiment, the operator started the two systems simultaneously. The experiments were limited to 100 seconds: during the first five seconds, subjects were in an upright position until the 6th second, when they did a first squat quickly. Then, they remained upright until the 50th second, then did a squat again to distinguish the state of tilting from that of being still upright; the subjects were instructed to tilt their bodies but not to step during the experiment. To minimize the human factor in the experiment, before the trial, the subjects were told the basic requirement and kept the action standardized. When standing still, the space between feet were the same as the width of the hips, the trunk was held stable, and the hands were allowed to droop on both sides of the body. When tilting, arms were held naturally on both sides of the body, and all subjects' trunk inclination angle was maintained at around $-15^{\circ}$ to $45^{\circ}$ in the anteroposterior and $20^{\circ}$ in the lateral direction. Regarding the accuracy of COG estimations, previous researchers did significant work on the accuracy of SKC or SAMP methods during static or balance recovery, as described in Refs. 1 and 2. The largest standard deviation (SD) in three directions is $8.3 \mathrm{~mm}$, and the mean tolerance in error in the $X, Y$, or $Z$ directions was held at around $2.4-5.8 \mathrm{~mm}$.

\section{Results}

The displacement of the COG was calculated on the basis of data from the capture system and the inclinometer system. All three directions were fundamentally similar in magnitude across the 20 tilt trials, as shown in Fig. 3.

The shape of the plots was similar for both the methods of motion capture and the inclinometer system. To prove the stability and precision of the inclinometer measurement system, the study compared the results from three aspects: extreme value, distribution trajectory, and root-meansquare error (RMS). The smallest RMS between the two detection methods in the directions occurred in the vertical $(Z)$ direction for tilt trials. In the anteroposterior $(X)$ direction, the RMS was the largest in the three directions. The mean RMS of the two systems in three directions is 


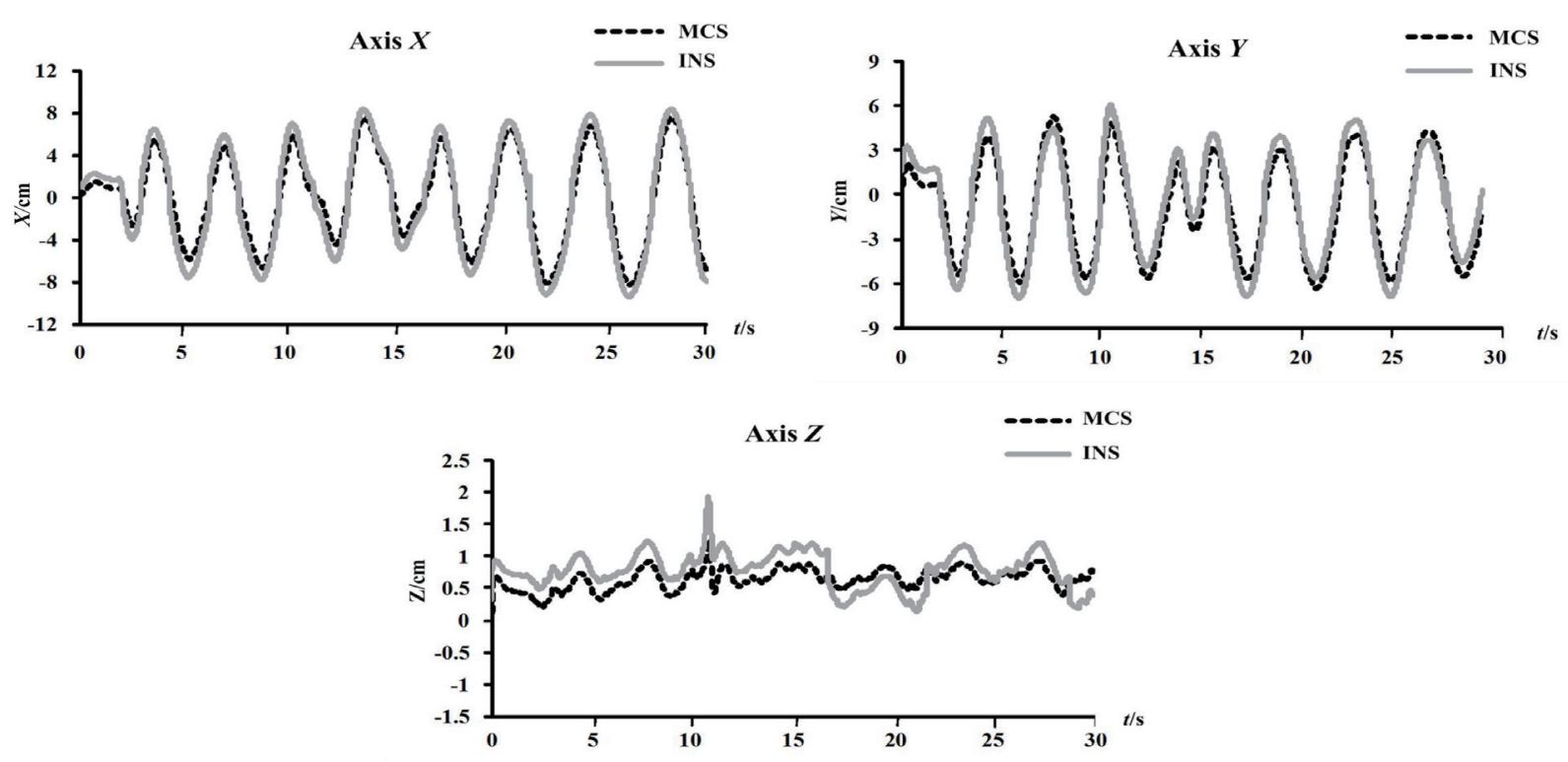

Fig. 3. Comparison of the trajectories of COGs from two different systems in 3D directions. The value of the ordinate represents the wave range in units of $\mathrm{cm}$. The value of the abscissa represents the collection time and in units of s. Dotted lines represent the results from the motion capture system; the solid lines represent the results from the inclinometer network.

$1.27-4.89 \mathrm{~cm}$, and the difference is within a range of $0.15-0.41 \mathrm{~cm}$, as shown in Table 1 . The range of the fluctuation in the COG trajectory is similar to the data from motion capturing system, and the degree of deviation between the two systems is low.

To compare the difference in the COG wave range between the two systems, we collected the maximum and minimum values of the COGs, the absolute error in the maximum and minimum values in three directions, and the mean and SD in Table 2.

As shown in Table 2, the COG deviation in the maximum and minimum values between the two systems is in the range of 0.01-1.09 cm. In addition, the mean SD compared with the values from the motion capture system in $3 \mathrm{D}$ directions is also small, in the range of $0.10-0.60 \mathrm{~cm}$. Compared with measurements in Refs. 1 and 2, this result lies in the range of tolerance for error. These data established the high measurement accuracy of inclinometer network systems.

\section{Discussion}

As found in the literature, the trajectories of COG from two different systems are similar to each other and show almost the same displacement of COG in three directions. The extreme values in three directions are also similar; the error and RMS values of the two systems' extreme values are small. All results establish that the estimations of COG position by the motion capture system using 38 markers and an inclinometer network are very close, as they are based on the same anthropometric data. Generally, the tolerable error in three directions is in the range of $0.10-0.60$ $\mathrm{cm}$, which is a reasonable range based on the results in Ref. 1. According to the results for the COG from the two different systems, the RMS is bigger in the $X$ direction than in the mediolateral 
Table 1

Analysis of the samples' distribution of COG in 3D directions. The RMS values from two different detection methods are shown in this table, along with the calculated mean of RMS and SD.

\begin{tabular}{|c|c|c|c|c|c|c|}
\hline & $X(\mathrm{~cm})$ & & $Y(\mathrm{~cm})$ & & $(\mathrm{cm})$ & \\
\hline & MCS & INS & MCS & INS & MCS & INS \\
\hline RMS & $0.05-1.09$ & $0.12-0.73$ & $0.05-0.82$ & $0.14-0.92$ & $0.01-0.48$ & $0.04-0.46$ \\
\hline Mean & 0.57 & 0.45 & 0.39 & 0.36 & 0.22 & 0.18 \\
\hline SD & 1.26 & 1.51 & 0.80 & 0.98 & 0.37 & 0.35 \\
\hline
\end{tabular}

Table 2

Comparison of the COG data from two systems and the deviation of extreme values in 3D directions, the absolute error, the mean of absolute error, and the SD.

\begin{tabular}{lcccccc}
\hline & $X(\mathrm{~cm})$ & \multicolumn{3}{c}{$Y(\mathrm{~cm})$} & \multicolumn{3}{c}{} \\
\cline { 2 - 7 } & MAX & MIN & MAX & MIN & MAX & MIN \\
\hline Absolute error & $2.05-6.38$ & $1.98-6.91$ & $1.34-4.18$ & $1.36-4.65$ & $0.71-2.11$ & $0.77-2.03$ \\
Mean & 4.48 & 4.90 & 2.79 & 3.08 & 1.27 & 1.42 \\
SD & 1.26 & 1.51 & 0.80 & 0.99 & 0.37 & 0.35 \\
\hline
\end{tabular}

$(Y)$ direction, and the RMS in the $Z$ direction is the smallest, which is consistent with the COG trajectory and the distribution of extreme values. These results illustrate that the wave range of COG in the $X$ direction is largest and the degree of dispersion in the $Z$ direction is smallest. The differences among the $X, Y$, and $Z$ directions are consistent with results reported in Refs. 13 and 14 . These references pointed out that during standing, the ankle is a core joint for regulating balance; this joints' regulating ability in the anteroposterior direction is stronger than that in the $Y$ direction.

Researchers have shown that the simplified segmental method using a sacral skin marker can accurately estimate COG trajectories. ${ }^{(15)}$ However, Ref. 8 indicates that this method has lower precision than the SIMP method, which is based on an analytical method. Therefore, in this model, the SKC method to estimate COM and for convenient calculation, the body is treated as consisting of five parts based on the Songjing model. Even though the simplified method is exact, the trial is still based on a motion capture system. Considering that it cannot be readily used in clinics or rehabilitation hospitals because of the complex and precise nature of this kind of system, we proposed a simplified method using an inclinometer network system that has advantages of light weight, compactness, and portability. It reduces experimentation and postprocessing time, is appropriate for studying stability in dynamic situations, and works well for healthy populations. It represents a trade-off between accuracy and simplicity, but this method would be useful to estimate COG positions during movement, particularly for balance analysis.

Even though the study showed the accuracy and precision of the 3D gravity center detection systems, the study used only 20 college students as a sample. Future studies will involve more representative volunteers, including the young, the old, and the disabled, to validate the practicality of this method and to apply it in a clinical setting as soon as possible. 


\section{Conclusions}

In this research, we proposed a method of detecting the 3D COG based on the Songjing model and involved the construction of the system. The characteristic of this system involves the introduction of an inclinometer network system to measure the inclination angle of combined segments in real time. The 3D coordinates of the COG can be obtained by substituting angles into a formula, which was convenient, cheap, and required fewer calculations. By comparing results to those from a motion caption system, we showed that the COG data obtained by the two systems were highly consistent, which confirmed the feasibility of the method. Therefore, this inclinometer network system could be used to estimate the balance function as a detection method.

\section{Acknowledgments}

This work was supported by a grant from the Key Laboratory of Digital Medical Engineering of Hebei Province, China, and the Natural Science Foundation of Hebei Province, China (No. H2016201201).

\section{References}

1 A. Mapelli, M. Zago, L. Fusini, and D. Galante: Gait \& Posture 39 (2014) 460.

2 E. M. Gutierrez-Farewik and A. Bartonek: Hum. Mov. Sci. 25 (2006) 238.

R. P. Cawsey, R. Chua, and M. G. Carpenter: Gait \& Posture 29 (2009) 280.

4 S. L. Tzedek, H. I. Krebs, J. E. Arle, and J. L. Shils: Exp. Brain Res. 86 (2011) 211.

5 T. P. Andriacchi, L. Muendermann, and S. Corazza: J. Biomech. 39 (2006) 190.

6 H. Chaudhry, B. Bukiet, and Z. Ji: J. Bodywork Mov. Therapies 15 (2011) 82.

7 D. B. Murillo, R. S. Solana, and F. J. Vera-Garcia: Hum. Movement. Sci. 31 (2012) 1224.

8 GB/T 17245-2004, Inertial Parameters of Adult Human Body (in Chinese).

9 R. Tisserand, T. Robert, R. Dumas, and L. Cheze: Gait \& Posture 48 (2016) 64

10 Q. L. Zhang, F. L. Chi, and Q. K. Luo: Chin J. Med. Instr. 25 (2001) 215.

11 L. Tesio, V. Rota, C. Chessa, and L. Perucca: J. Biomech. 43 (2010) 938.

12 M. Goffredo, M. Schmid, S. Conforto, and T. D. Alwssio: Med. Eng. Phys. 28 (2006) 719.

13 L. Pei, H. Li, and Y. Fu: J. Med. Biomech. 27 (2012) 444.

14 L. Tesio, V. Rota, and C. Chessa: J. Biomech. 43 (2011) 938.

15 Y. Feng and Y. Pai: J. Biomech. 47 (2014) 3087. 